\title{
Quantitative versus Qualitative Ansätze in der Transformationsforschung
}

\author{
Klaus Lindert ${ }^{*}$
}

(1) Das Realphänomen der Transformation und die Frage nach der Angemessenheit und Logik der Forschungsansätze konfrontiert die Managementwissenschaft einerseits mit hohen Erwartungen an praktische Problemlösungen und andererseits mit den möglicherweise hochgesteckten erkenntnis- und wissenschaftstheoretischen Erwartungen an generelle Aussagesysteme (vgl. Lakatos 1982; Popper 1994). Die zyklisch wiederkehrenden Kontroversen über das Theorie-Praxis-Problem haben vielfältige wissenschaftstheoretische Fragen aufgeworfen, aber bisher zu keiner zufriedenstellenden oder überzeugenden Lösung geführt (vgl. Albert 1982; Chmielewicz 1994; Wunderer 1995). Der eigene Ansatz orientiert sich an der ursprünglich Lewin zugeschriebenen Aussage: 'Nothing is supposed to be as practical as a good theory, but nothing seems more impractical than a bad one' (Bass 1990: 37). Die Praxis erwartet Informationen über Gestaltungsmöglichkeiten. Aber erst aus dem Wissen über Wirkungsmechanismen können durch tautologische Transformation Ursache-Wirkungs-Beziehungen in Zweck-Mittel-Beziehungen überführt werden. Gestaltung ist durchaus ohne Erkenntnis möglich, aber eine so verstandene Gestaltungstechnik (Sozialtechnik) würde sich auf dem Niveau einer handwerklichen Kunstlehre befinden (Schanz 1988: 38). Als im Jahre 1988 die Forschungsstrategie für ein empirisch-vergleichendes Projekt zum betrieblichen Management mit Partnern aus Osteuropa festgelegt wurde, standen die Fragen des graduellen Umbaus der osteuropäischen Wirtschaft und des Aufbaus neuer Handlungsmuster in unterschiedlichen Dimensionen im Vordergrund. Der Forschungsansatz entstammt der komparativen Managementforschung, die sich auf den Vergleich von Handlungssystemen in unterschiedlichen Ländern, Kulturkreisen und politisch-ökonomischen Systemen konzentriert. Das zentrale Interesse besteht in der Identifikation spezifischer und universeller Problemlösungsprozesse im Management. Das Erkenntnisinteresse richtet sich auf Führungskräfte als Akteure betrieblicher Transformationsprozesse. Der Wandel und/oder

* Klaus Lindert, geb. 1958, Dr. rer. pol., Akademischer Rat an der Technischen Universität Braunschweig, Institut für Wirtschaftswissenschaften, Abt. Unternehmensführung, Arbeitsschwerpunkte: Personalwirtschaft, Internationales Management. 
Untergang ökonomischer, rechtlicher und sozialer Institutionen auf der einen Seite und das Fehlen eines kohärenten Ideensystems auf der anderen Seite reaktiviert auf der individuellen Ebene der betrieblichen Entscheidungsträger implizite Referenzmuster zum Mitteleinsatz und zur Zielformulierung unter Unsicherheit. Im Forschungsansatz erhält das kognitive Wertkonzept daher eine zentrale Bedeutung. Werte können als selbstreferentielle Orientierungsmuster sozialer Systeme aufgefaßt werden. Im Rahmen einer Längsschnitterhebung in den Jahren von 1989 bis 1994 konnten in Deutschland (BRD/DDR/neue Länder), Frankreich, Irland, Polen, Tschechien, Rußland, Ukraine, Georgien, Estland, Bulgarien und Albanien 2237 Führungskräfte zu Führungskonzeptionen und dem Selbstverständnis als Führungskraft befragt (Direkterhebung, strukturiertes Interview und qualitative Einzelerhebung mit Fallbeschreibungen) werden (vgl. Lindert 1993; 1996). Aussagen zum Wandel von Führungskonzeptionen sowie den Phasen und Formen der länderspezifischen Anpassung erscheinen damit möglich. Der eigene Ansatz wird von der Auffassung getragen, daß sich menschliches Handeln auf genotypische Äquivalenzklassen begründet. Sozialwissenschaftliche Aussagen können damit einen allgemeingültigen Status aufweisen. Ausgehend von den erfahrungswissenschaftlichen Erkenntnissen der Organisations- und Führungsforschung hat der extreme 'Situationalismus' in eine forschungsstrategische Sackgasse geführt. Die phänotypische Variabilität ist das Produkt kontingenter Anreize und moderierender situativer Effekte. Raumzeitlich beschränkt einigen sich Individuen über einen vorläufigen Bezugsrahmen, der ein konsensuelles Handeln ermöglicht. Die länder-(kultur-) spezifische Variabilität von Verhalten ist damit ein gültiges Produkt unterschiedlicher Realitätsbearbeitung und Problemlösung.

(2) Faßt man als Erfahrungsobjekt der Transformationsforschung den gesamten Prozeß des Übergangs von einer zentral geleiteten Planwirtschaft zur (sozialen) Marktwirtschaft auf, so ist durch den Problembezug ein disziplinäres Erkenntnisobjekt nur schwer zu isolieren. Neben der geplanten Transformation der Wirtschaftsordnung und des Rechtssystems vollzieht sich ein politischer, sozialer und kultureller Wandel mit geplanten, ungeplanten, reproduktiven und regenerativen Aspekten in länderspezifischer Anordnung und Ausformung. In den letzten Jahren ist eine Fülle von Herausgeberpublikationen und Zeitschriftensonderbänden zur Transformation in Osteuropa erschienen, in denen das Problemfeld interdisziplinär und praxisorientiert bearbeitet wird. Die Erkenntnisse bleiben aber aufgrund einer fehlenden Theorie der Transformation fragmentarisch. Witt (1993: 1157) hebt als vordringliche Aufgabe der Transformationsforschung die Dokumentation des vielschichtigen Transformationsprozesses hervor. Aus der Makroperspektive wird versucht, die anfänglichen Spekulationen über gangbare Transformationspfade (Strategien) hinsichtlich der Angemessenheit der Wirkungen zu überprüfen. Es liegen zum 
Teil komparative makroökonomische Daten zum Wachstum, der Stabilität und Wohlfahrtsentwicklung vor, die den Wandel der letzten fünf Jahre dokumentieren und erste Prognosen über die Transformationsökonomien ermöglichen (vgl. Kosta 1995: 243ff.). Auf der Ebene der betrieblichen Transformation stehen die Fragen der grundlegenden Sanierung und Restrukturierung von Unternehmen, der Rekapitalisierung und der Verfügungsrechte/Eigentümerkontrolle im Vordergrund (vgl. u.a. Albach 1993). Die managementorientierte Transformationsliteratur konzentriert sich noch auf Prozeßbeschreibungen, die Rekonstruktionen vergangener Zustände und die Dokumentation von Erfahrungen und subjektivem Erleben von Betroffenen. Zukünftige Anforderungen, Potentialabschätzungen, das Rollenverständnis und Unterschiede zwischen Führungskräften sowie länderübergreifende Einschätzungen werden beschrieben (vgl. u.a. v. Rosenstiel 1994). Im Rahmen ideographischer Fallstudien werden länder-, regional- und branchenspezifische Besonderheiten und Probleme dokumentiert. Es sind deskriptive Aussagen über raumzeitlich beschränkte, singuläre Ereignisse. Obwohl der Prozeßbegriff verwendet wird, werden wiederholte Beobachtungen im Schwerpunkt nicht dokumentiert.

(3) Ökonomischer, politischer und kultureller Wandel des Koordinationsmechanismus der Austauschprozesse vollzieht sich nicht im sozialen Vakuum. Aktuell besteht wahrscheinlich kein Zweifel, daß besonders die zukünftigen Entscheidungsträger in Wirtschaft und Verwaltung/Politik die Hauptlast der Transformation $\mathrm{zu}$ tragen haben. Kontroversen und Zweifel bestehen hinsichtlich der technologischen Aussagen über die einzusetzenden Mittel. Für die Bestimmung individueller Entscheidungen dürften kognitive und normative Überzeugungstatbestände in der individuellen Problemsicht von erheblicher Bedeutung sein (vgl. Albert 1994: 139). Die Vergangenheit hat gezeigt, daß nicht nur finanzielle Mittel, sondern auch transferiertes Wissen und andere Ressourcen zum Teil ungenutzt blieben oder nicht die erwünschten nachhaltigen Effekte erzielten. Der anfängliche Enthusiasmus des politischen Umbruchs mit hohen Erwartungen an einen wirtschaftlichen Aufschwung ist dem Realismus gewichen. Dieser Realismus könnte den Anlaß geben, existierende ZweckMittel-Behauptungen auf ihren faktisch-empirischen Wahrheitsgehalt $\mathrm{zu}$ überprüfen. Formal bedeutet die Behandlung des Transformationsphänomens für den strengen Empiristen die wiederholte Beobachtung von Elementen und Relationen im Objektbereich. Damit entstehen die Fragen nach dem inhaltlich interessierenden Objektbereich und der Aggregationsstufe der Beobachtung. Ohne theoretisches Gerüst ist nicht entscheidbar, welche Variablen gestaltet, beobachtet und dokumentiert werden müssen. Während einerseits von Bausteinen einer Makrotheorie der Transformation gesprochen wird (vgl. Herr/Westphal 1993), steht auf der anderen Seite die Schaffung einer Realtheorie betrieblicher Transformation im Vordergrund (vgl. Witt 1993). Internationale Vergleiche zeigen, daß makroökonomische Erklärungsmodelle 
wichtige Gestaltungsparameter liefern, aber nur bedingt den Aufstieg und Niedergang wirtschaftlicher Institutionen und Länder prognostizieren können. Die Leistungsfähigkeit eines Landes ist das Resultat konkurrenzfähiger Unternehmen und Branchen. In der Managementliteratur nehmen Begriffe wie Transformation, Wandel, Fortschritt, Wachstum und kontinuierliche Verbesserung einen breiten Raum ein (vgl. u.a. Gomez/Müller-Stewens/Wunderer 1994). Ein Mangel an theoretischen Bezugsrahmen und konkurrierenden Hypothesen besteht nicht (vgl. Krüger 1994; Steinle 1985). Der wissenschaftliche Weg besteht aber darin, die Wirkung von betriebswirtschaftlichen Instrumenten in unterschiedlichen Kontexten und die modifizierende Rückwirkung der Kontextbedingungen auf die Ausgestaltung der Instrumente im Rahmen einer inhaltlich präzisierten Weg-Ziel-Analyse (Wirkungsanalyse) zu bestimmen. In aktueller Problemsicht scheint auch die Tragfähigkeit eines Transformationskonzeptes von einer erkennbaren Weg-Ziel-Definition abzuhängen. Unter ökonomischer Erkenntnisperspektive besteht das Transformationsziel in der Einkommenserzielung im gesamten betrieblichen Netzwerk. Ein operationaler Weg könnte sich zunächst auf die Bündelung, Koordinierung und Prozeßbegleitung lokaler Aktivitäten zur Initialisierung dezentral gesteuerter, überlebensfähiger Strukturen beschränken.

(4) Die methodische Kritik bezieht sich auf die Versuche, die Transformation (den Zustandsübergang) zeitpunktbezogen im Querschnitt zu erfassen (z.B. >X ist jetzt stärker ausgeprägt als früher $<$ oder $>Y$ wird in Zukunft mehr erforderlich sein als jetzt $<$ ). Eine retrospektive Datenaufnahme ist aus empirischer Perspektive aufgrund kognitiver Verzerrungen in der Rekonstruktion vergangener Sachverhalte nicht zu vertreten. Selbst die Einbeziehung scheinbar objektiv dokumentierter Vergangenheitsdaten führt zum erkenntnistheoretischen Problem der Rekonstruktion der Erhebungsbedingungen. Auch die dokumentierten projektiven Entwürfe zukünftiger Zustände sind auf die Auswirkungen zu überprüfen. Die Angemessenheit der Forschungsmethodologie im Bereich der Organisationsund Managementforschung hat in den letzten Jahren eine zunehmende Beachtung gefunden. Speziell die länder- und kulturübergreifende Managementforschung (Comparative/Cross-Cultural Management Research) hat die Sensitivität auf die Forschungsmethode gelenkt (vgl. Jean Lee 1992: 87). Dabei steht die Frage im Vordergrund, ob sozialwissenschaftliche Phänomene innerhalb des naturwissenschaftlichen Forschungsparadigmas erforscht werden können (vgl. Topitsch 1993). Polarisiert man quantitative (naturwissenschaftliche, objektive, positivistische, universelle) und qualitative (sozialwissenschaftliche, subjektive, phänomenologische, partielle) Methoden, so wird in der managementorientierten Transformationsforschung ein Trend zu Fallstudien und qualitativen Methoden sichtbar. Zweifellos bestimmt die Auswahl der jeweiligen Methode den Erkenntnisvorgang. Problematisch ist, daß 
die Hermeneutik, die das Verstehen von Sinnzusammenhängen vor die Erklärung stellt, selbst ein Objekt der Erklärung darstellt. Bedeutung gewinnen verstehende Methoden im Entdeckungszusammenhang und zur unterstützenden qualitativen Interpretation quantitativer Daten. Auf ökonomische Fragestellungen bezogen haben die Mängel der neoklassischen Analyse 'nichts mit der Benutzung der Methode der nomologisch gestützten Erklärung zu tun und geben daher keinen Anlaß, aus methodologischen Gründen eine hermeneutische Wende in den Sozialwissenschaften zu befürworten' (Albert 1994: 149).

(5) Aussagen über Forschungspotentiale in Osteuropa sind nur auf der Basis von Erfahrungswerten möglich. $\mathrm{Zu}$ Beginn des Transformationsprozesses ermöglichte die Existenz zentraler Forschungsinstitutionen eine relativ gute Forschungskooperation mit Ökonomen und Soziologen. Das Forschungspotential und auch die Ressourcen waren gegeben. Diese zentralen Institutionen wurden im Verlauf der Transformation aufgelöst oder restrukturiert. Die personellen Kapazitäten wurden erheblich reduziert. Der Verlust an Forschungskapazität wurde durch die Möglichkeiten alternativer Einkommenserzielung unterstützt. Aktuell werden die personellen Kapazitäten an den Universitäten durch die Einführung der neuen wirtschaftswissenschaftlichen Studiengänge, die Transformation der Lehrinhalte und die hohe Studiengangnachfrage gebunden. Die Aktivierung von Forschungspotentialen und der Zugriff auf universitäre Forschung ist daher häufig von externen Anreizen und dem Transfer von Mitteln abhängig.

(6) Technologische Aussagen, wie die Transformation wird durch den Transfer von Managementwissen unterstützt, lassen sich zu Hypothesenbündeln reformulieren und empirisch überprüfen. Da die aktuellen praktischen Probleme raumzeitlich gebunden existieren und die Forderungen nach deduktiv-nomologischen Erklärungsmodellen in den Sozialwissenschaften praktisch nicht zu erfüllen sind, stellt sich die Frage nach dem methodologischen Prinzip. Als geeignetes Erklärungsmodell erscheint die Propensitätsinterpretation der Wahrscheinlichkeit von Popper. 'Die Propensität ist die Verwirklichungstendenz eines Dings, in einer bestimmten Situation eine bestimmte Eigenschaft oder einen bestimmten Zustand einzunehmen' (Popper/Eccles 1982: 48). Die Maßnahmen (z.B. Mittel der Transformation oder Unternehmensführung) stellen nur unvollständige Ursachen der Zielverwirklichung dar. Eine kausale Beziehung ist ein Sonderfall der Propensität. Akzeptiert man die PropensitätsErklärung als Begründung situativer Studien in den Sozialwissenschaften, dann ist die Verwirklichungstendenz menschlichen Handelns (Entscheidens) nur aus der Situationslogik moderierender Faktoren (Bedingungen) erklärbar. Nach dem Propensitäts-Ansatz ist zunächst nach den Klassen von Verwirklichungsoptionen zu fragen. Auf individueller Ebene könnte dieses die generelle Lernfähigkeit betreffen. Im Aggregat könnte die zeitliche 
Verzögerung im Transformationsprozeß ganzer Gesellschaften erklärt werden. Der zweite Aspekt betrifft die Erfassung der Situationslogik, die zur Beeinflussung der Verwirklichung beiträgt. Da das zu beobachtende Objekt der Transformationsprozeß selbst ist, sind Daten im zeitlichen Längsschnitt zu beobachten. Mit Hilfe eines Strukturgleichungsmodells, das die multiplen theoretischen Behauptungen enthält, könnte der Prozeß in seiner Gestalt (FitKonzept) simultan auf empirischen Gehalt überprüft werden. Der Kern des quasi-experimentellen Designs besteht aus absichtlichen und planmäßigen Einwirkungen auf die Entstehung und den Verlauf der Beobachtung. Diese multikausalen Gestaltungsvariablen sind strikt von den intervenierenden Unternehmens- oder Personenattributen und den multiplen moderierenden Kontingenzvariablen zu trennen. Konkret bedeutet dieser Mehrebenen-Ansatz eine systematische Überprüfung und strategische Kontrolle der logischen Struktur von Maßnahmenbündeln hinsichtlich der Nachhaltigkeit der Auswirkungen und Zielbeiträge auf regionalpolitischer, unternehmenspolitischer und individueller Ebene im Rahmen etablierter internationaler Wirtschaftsförderungsprogramme.

\section{Literatur}

Albach, H. (1993): Analyse des Transformationsprozesses in den neuen Bundesländern. In: Zeitschrift für Betriebswirtschaft, Unternehmen in den neuen Bundesländern, Erfahrungen mit Transformationsprozessen. Ergänzungsheft 1. VII-VIII.

Albert, H. (1982): Die Wissenschaft und die Fehlbarkeit der Vernunft. Tübingen: Mohr.

Albert, H. (1994): Kritik der reinen Hermeneutik: Der Antirealismus und das Problem des Verstehens. Tübingen: Mohr.

Bass, B.M. (1990): Bass \& Stogdill's Handbook of Leadership. Theory, Research, and Managerial Application. 3.Aufl. New York u.a.: Free Press.

Chmielewicz, K. (1994): Forschungskonzeptionen der Wirtschaftswissenschaften. 3. unveränd. Aufl. Stuttgart: Schäffer-Poeschel.

Gomez, P. / Hahn, D. / Müller-Stewens, G.; Wunderer, R. (Hrsg.) (1994): Unternehmerischer Wandel - Konzepte zur organisatorischen Erneuerung. Wiesbaden: Gabler.

Herr, H. / Westphal, A. (Hrsg.) (1993): Transformation in Mittel- und Osteuropa: Makroökonomische Konzepte und Fallstudien. Frankfurt a.M.: Campus.

Jean Lee, S.K. (1992): Quantitative versus Qualitative Research Methods: Two Approaches to Organisation Studies. In: Asia Pacific Journal of Management. 9. 1. pp. 87-94.

Kosta, J. (1995): Die Systemtransformation in den CEFTA-Ländern: Strategien und Ergebnisse. In: Osteuropa-Wirtschaft. 40. 3. pp. 238-247.

Krüger, W. (1994): Transformations-Management. In: Gomez, P. / Hahn, D. / Müller-Stewens, G. / Wunderer, R. (Hrsg.): Unternehmerischer Wandel, Wiesbaden: Gabler. S. 199-228. 
Lakatos, I. (1982): Die Methodologie der wissenschaftlichen Forschungsprogramme. Braunschweig. Wiesbaden: Vieweg.

Lindert, K. (1993): Führungskonzeptionen auf der Basis impliziter Wertorientierungen - Gemeinsamkeiten und Unterschiede bundesdeutscher und mittel- und osteuropäischer Führungskräfte. Stuttgart: Metzler \& Poeschel.

Lindert, K. (1996): Führungskonzeptionen im Wandel: Eine interkulturelle und intertemporale Studie. In: Lang, R. (Hrsg.): Wandel von Organisationskulturen in Ostdeutschland und Osteuropa. München, Mering: Hampp. (im Druck).

Popper, K. (1994): Logik der Forschung. 10. Aufl. Tübingen: Mohr.

Popper, K.R. / Eccles, J.C. (1982): Das Ich und sein Gehirn. München, Zürich: Piper.

Rosenstiel, L.v. (Hrsg.) (1994): Führung im Systemwandel, Untersuchungen zum Führungsverhalten beim Übergang von der Plan- in die Marktwirtschaft. München: Hampp.

Schanz, G. (1988): Erkennen und Gestalten, Betriebswirtschaftslehre in kritisch-rationaler Absicht. Stuttgart: Poeschel.

Steinle, C. (1985): Organisation und Wandel, Konzepte - Mehr-Ebenen-Analyse (MEA) Anwendungen. Berlin u.a.: de Gruyter.

Topitsch, E. (Hrsg.) (1993): Logik der Sozialwissenschaften. Frankfurt a.M.: Hain.

Witt, C.D. (1993): Wandel der Aufbauorganisation im Transformationsprozeß ostdeutscher Industrieunternehmen. In: Zeitschrift für Betriebswirtschaft. 63. 11. S. 1157-1168.

Wunderer, R. (Hrsg.) (1995): Betriebswirtschaftslehre als Management- und Führungslehre. 3. überarb. u. erg. Aufl. Stuttgart: Schäffer-Poeschel. 